\title{
Chronic kidney disease and diabetes associated with long-term outcomes in patients receiving percutaneous coronary intervention
}

Mao-Jen Lin ${ }^{1,2}$, Jung Lee ${ }^{3,4}$, Chun-Yu Chen ${ }^{5,6}$, Chia-Chen Huang ${ }^{7}$ and Han-Ping Wu U $^{3,4^{*}}$ (D)

\begin{abstract}
Background: The effect of diabetes mellitus (DM) and chronic kidney disease (CKD) on long-term outcomes in patients receiving percutaneous coronary intervention $(\mathrm{PCl})$ is unclear.

Methods: A total of 1394 patients who underwent $\mathrm{PCl}$ were prospectively enrolled and divided into 4 groups according to the presence or absence of DM or CKD. Baseline characteristics, risk factors, medications, and angiographic findings were compared. Determinants of long-term outcomes in patients undergoing $\mathrm{PCl}$ were analyzed.

Results: Patients with DM and CKD had the highest all-cause mortality and cardiovascular mortality (both $P<0.01$ ) but there were no differences existed in myocardial infarction (MI) or repeated $\mathrm{PCl}$ among the 4 groups $(P=0.19, P=0.87$, respectively). Patients with DM and CKD had the lowest even-free rate of all-cause mortality, cardiovascular mortality, $\mathrm{Ml}$, and repeated $\mathrm{PCl}(P<0.001, P<0.001, P<0.001$, and $P=0.002$, respectively). In the Cox proportional hazard model, patients with both DM and CKD had the highest risk of all-cause mortality (HR: 3.25, 95\% Cl: 1.85-5.59), cardiovascular mortality (HR: 3.58, 95\% Cl: 1.97-6.49), MI (HR: 2. 43, 95\% Cl: 1.23-4.08), and repeated PCl (HR: 1.79, 95\% Cl: 1.33-2.41). Patients with CKD alone had the second highest risk of all-cause mortality (HR: 2.04, 95\% Cl: 1.15-3.63), cardiovascular mortality (HR: 2.13, 95\% Cl: 1.13-4.01), and repeated PCl (HR: 1.47, 95\% Cl: 1.09-1.97).
\end{abstract}

Conclusions: DM and CKD had additive effect on adverse long-term outcomes in patients receiving PCl; CKD was a more significant adverse predictor than DM.

Keywords: PCl, Diabetes mellitus, Chronic kidney disease

\section{Background}

Percutaneous coronary intervention (PCI) is common in patients with coronary artery disease(CAD). However, a lot of risk factors will affect the outcome after patients receiving PCI. Diabetes mellitus (DM) is a major risk factor that affects outcomes in CAD patients undergoing PCI [1-3]. Recently, chronic kidney disease (CKD) has

\footnotetext{
*Correspondence: arthur1226@gmail.com

${ }^{3}$ Division of Pediatric General Medicine, Department of Pediatrics, Chang

Gung Memorial Hospital at Linko, No. 5, Fu-Hsin Street, Kweishan, Taoyuan, Taiwan

${ }^{4}$ College of Medicine, Chang Gung University, Taoyuan, Taiwan

Full list of author information is available at the end of the article
}

emerged as a risk factor in terms of outcomes in patients undergoing PCI [4-7].

The impact of DM and CKD on outcomes has been well studied in patients with acute coronary syndrome (ACS) undergoing PCI. Presence of CKD could affect long-term outcomes in patients with ST-elevation myocardial infarction (STEMI) managed by primary PCI and in-hospital mortality in patients with non-ST elevation myocardial infarction (non-STEMI) undergoing PCI $[8,9]$. After undergoing PCI, diabetic patients suffered from ACS had worse short-term and mid-term outcomes than non-diabetic patients with ACS [10-12]. As for patients with stable CAD who underwent PCI, 
diabetes was still an adverse predictor of mid-term outcomes.

For ACS patients with both DM and CKD, the coexistence of $\mathrm{DM}$ and CKD appeared to have the higher risk of MACE than DM alone or CKD alone [13]. However, the combined effect of DM and CKD on long-term prognosis in patients undergoing PCI is still unclear. Our aim was to clarify and compare long-term outcomes among four groups of patients: patients with both DM and CKD, with only DM, with only CKD, and without CKD and DM. We further analyzed the adverse predictors of clinical outcomes among these 4 groups.

\section{Methods}

\section{Study population}

A prospective cohort design using was conducted via medical records review from May 2007 to Dec 2014. The institutional review board and ethics committee approved the study protocol and monitoring for the study.

We consecutively recruited patients aged 30 to 90 years who were to undergo PCI from the inpatient clinic at the Taichung Tzu Chi Hospital, Taiwan. According to isolated or coexistence of risk factors, the patients were divided into four groups: patients without DM or CKD, patients with DM alone, patients with CKD alone, and patients with both DM and CKD. Patients with a scheduled PCI and previous history of malignancy were excluded. Most patients obtained regular follow-up in the outpatient department (OPD). For some patients who were lost to follow-up, usually a phone call was used to contact the patients themselves or their families. A survey of cardiovascular mortality (CV mortality), all-cause mortality, myocardial infarction (MI), and repeated PCI procedures was completed at the end of the study.

\section{Data gathering and analysis}

Baseline characteristics including body habitus, biochemical data, angiographic findings from cardiac catheterization, exposed risk factors, and variant therapeutic strategies such as drug medications and invasive procedures (balloon angioplasty, bare metal stent deployment, or drug-eluting stent deployment) were all obtained for our study. Diabetes was defined as a fasting plasma glucose level of $>126 \mathrm{mg} / \mathrm{dL}$, a casual plasma glucose level of $>200 \mathrm{mg} / \mathrm{dL}$, or a hemoglobin A1c (HbA1c) level of $>6.5 \%$ in this study [14]. Estimated glomerular filtration rate (eGFR) and chronic kidney disease (CKD) stage were divided into 5 stages: stage l, eGFR $\geq 90 \mathrm{~mL} / \mathrm{min} / 1.73 \mathrm{~m}^{2}$; stage 2 , eGFR of $60-89 \mathrm{~mL} / \mathrm{min} / 1.73 \mathrm{~m}^{2}$; stage 3 , eGFR of $30-59 \mathrm{~mL} / \mathrm{min} / 1.73 \mathrm{~m}^{2}$; stage 4 , eGFR of $15-29 \mathrm{~mL} /$ $\mathrm{min} / 1.73 \mathrm{~m}^{2}$; and stage 5 , eGFR of $<15 \mathrm{~mL} / \mathrm{min} /$ $1.73 \mathrm{~m}^{2}$ or dialysis. CKD was defined as an eGFR of $<60 \mathrm{~mL} / \mathrm{min} / 1.73 \mathrm{~m}^{2}$, which was equal to or greater than CKD stage 3, in this study [15]. Hypercholesterolemia was defined as a serum cholesterol level of $>200 \mathrm{mg} / \mathrm{dL}$ or an LDL-C level of $>100 \mathrm{mg} / \mathrm{dLF}$ or the angiographic and hemodynamic data, we measured the central aortic pressure (CAP) and left ventricular ejection fraction (LVEF). The CAP was obtained by using a pigtail catheter while performing a coronary angiography. Angiographic findings including number of diseased vessels and lesion locations were calculated, and lesion severity and complexity were evaluated via the Synergy between PCI with Taxus and cardiac surgery score (Syntax Score) [16]. The left ventricular ejection was estimated through angiographic ventriculography or stress ventriculography.MI was defined as an MI attack after index PCI, accompanied by a 3-fold elevation in cardiac enzymes from the baseline value. General characteristics, major risk factors, angiographic findings, PCI strategies were analyzed, The primary end-points including all-cause death, cardiovascular death, myocardial infarction and repeated PCI were also compared among the 4 groups. The beginning of follow-up was the date of index PCI, and the duration of follow up was from the beginning through June 30, 2015 or if any of above primary end-points happened.

\section{Statistical analysis}

The analysis was primarily used to assess differences among the 4 groups. Analysis of variance (ANOVA) was used for test continuous variables, and chi-square test was used to test categorical variables. Log-rank test and Kaplan-Meier curves were used to compare survival differences. Cox proportional hazards model was used to test the effect of independent variables on hazards. $P$ values of $<0.05$ were considered significant. All analyses were performed using the statistical package SPSS for Windows (Version 23.0 SPSS Inc., Chicago, IL, USA).

\section{Results}

During the whole study period, a total of 1394 patients who underwent a successful PCI procedure were enrolled. Among them, 509 patients in the control group had neither DM nor CKD, 254 patients had DM alone, 320 patients had CKD alone, and 311 patients had both $\mathrm{DM}$ and CKD. There were no differences in mean follow up time among the 4 groups (control group, $31.7 \pm 22.6$ months; DM alone, $31.1 \pm 22.4$ months; CKD alone, $23.8 \pm 19.7$ months; and both DM and CKD, $23.0 \pm 19.2$ months; $P=0.08$ ).

General characteristics are listed in Table 1. Patients with CKD alone and with both DM and CKD were much older than patients in the other groups $(P<0.01)$. As for body habitus parameters, patients with CKD alone and patients with both DM and CKD had a lower 
Table 1 General Characteristics of the Study Population

\begin{tabular}{|c|c|c|c|c|c|}
\hline \multirow[t]{2}{*}{ Variable } & \multicolumn{4}{|l|}{ Study Groups } & \multirow[t]{2}{*}{$P$ value } \\
\hline & Control $(N=509)$ & DM alone $(N=254)$ & CKD alone $(N=320)$ & $\mathrm{DM}$ and $\mathrm{CKD}(N=311)$ & \\
\hline Age (y) & $58.1 \pm 10.8$ & $59.6 \pm 10.3$ & $72.2 \pm 10.1$ & $68.6 \pm 10.3$ & $<0.01$ \\
\hline Weight (kg) & $70.9 \pm 11.8$ & $72.2 \pm 14.4$ & $62.0 \pm 12.0$ & $64.6 \pm 12.0$ & $<0.01$ \\
\hline Height (cm) & $164.5 \pm 8.0$ & $163.6 \pm 8.8$ & $159.6 \pm 7.9$ & $160.2 \pm 8.4$ & $<0.01$ \\
\hline BMI $\left(\mathrm{kg} / \mathrm{m}^{2}\right)$ & $26.2 \pm 3.6$ & $26.9 \pm 4.3$ & $24.3 \pm 3.9$ & $25.1 \pm 3.8$ & $<0.01$ \\
\hline CSP & $133.4 \pm 20.7$ & $135.8 \pm 23.0$ & $136.5 \pm 25.3$ & $143.1 \pm 26.3$ & $<0.01$ \\
\hline CDP & $76.0 \pm 12.3$ & $73.8 \pm 13.1$ & $70.7 \pm 13.1$ & $70.3 \pm 13.2$ & $<0.01$ \\
\hline Cholesterol (mg/dL) & $184.4 \pm 43.5$ & $180.3 \pm 45.4$ & $177.0 \pm 42.2$ & $169.5 \pm 46.6$ & $<0.01$ \\
\hline $\mathrm{HDL}(\mathrm{mg} / \mathrm{dL})$ & $39.4 \pm 15.9$ & $36.1 \pm 14.7$ & $43.2 \pm 17.1$ & $37.6 \pm 15.4$ & $<0.01$ \\
\hline TG (mg/dL) & $160.0 \pm 105.0$ & $171.5 \pm 117.1$ & $134.9 \pm 94.1$ & $161.2 \pm 110.1$ & $<0.01$ \\
\hline LDL (mg/dL) & $113.0 \pm 39.7$ & $109.6 \pm 36.7$ & $106.9 \pm 36.3$ & $99.7 \pm 38.2$ & $<0.01$ \\
\hline Serum creatinine $(\mathrm{mg} / \mathrm{dL})$ & $0.9 \pm 0.2$ & $1.0 \pm 0.3$ & $2.2 \pm 2.4$ & $3.2 \pm 3.3$ & $<0.01$ \\
\hline
\end{tabular}

$D M$ alone diabetes alone, $C K D$ alone chronic kidney disease alone, DM and CKD both DM and CKD, BMI body mass index, CSP central aortic systolic pressure, CDP central aortic diastolic pressure, $H D L$ high-density lipoprotein cholesterol, $L D L$ low-density lipoprotein cholesterol, $T G$ triglyceride

body mass index(BMI), as compared with patients in the other 2 groups $(P<0.01)$. As for the hemodynamic parameters, patients with both DM and CKD had the highest central systolic pressure (CSP) and lowest central diastolic pressure (CDP), as compared with patients in the other groups $(P<0.01)$. As for baseline biochemistry, patients with both DM and CKD had the lowest cholesterol, low density lipoprotein-cholesterol(LDL-C) levels, and the poorest renal function (all $P<0.01$ ).

Demographic data for the study population are shown on Table 2. Female and hypertension preponderance were observed in patients with DM and CKD (both $P<0.01$ ); they also had the highest prevalence of stroke history $(P<0.01)$. However, patients with DM and CKD had the lowest prevalence of hypercholesterolemia and were the least likely to be current smokers (both $P<0.01)$. In terms of medication after PCI, we found that patients with CKD alone had the lowest aspirin use $(P<0.01)$ and highest diuretic use $(P<0.01)$. Patients with both DM and CKD had the lowest usage of angiotensin-converting enzyme inhibitors (ACEI) and statins $(P=0.02$ and $P<0.01$, respectively).

The angiographic findings and clinical outcomes are shown in Table 3. From the angiographic findings, double-vessel and triple-vessel disease were found more frequently in patients with both DM and CKD $(P<0.01)$. There were no differences in invasive strategy among the 4 groups $(P=0.34)$. As for adverse outcomes, patients with both DM and CKD had the highest allcause mortality and cardiovascular mortality rate (both $P<0.01)$; however, there was no difference in MI or repeated PCI rate among the 4 groups $(P=0.09$ and $P=0.32$, respectively). Figure 1 reveals the cumulative rate of freedom from MI, cardiovascular death, all-cause mortality, and repeated PCI among the 4 groups. Freedom from MI, all-cause mortality, CV death, and repeated PCI were lowest in the combined DM and CKD group $(P=0.002, P<0.001, P<0.001$, and $P<0.001$, respectively)

An outcome analysis and outcome predictors from the Cox proportion hazard model for MI, all-cause mortality, CV death, and repeated PCI are listed in Table 4. Patients with both DM and CKD carried the highest risk, as compared with the control group in terms of MI, CV death, all-cause mortality, and repeated PCI (hazard ratio [HR]: 2.43, 3.25, 3.58, and 1.79, respectively; all $P<0.01$ ). Based on the results from the Cox proportional hazard model, we found that previous MI and Syntax scores were predictors of MI (HR: 3.14 and 1.03 , respectively), and statin use could reduce the risk of MI (HR: 0.46). Age, previous MI, stroke history, and Syntax score were predictors of all-cause death (HR: $1.04,3.95,1.97$, and 1.03, respectively), beta blockers (BB) and statin use could reduce the risk (HR: 0.61 and 0.37, respectively). Previous MI, P2Y12 inhibitor use, and Syntax score were all predictors for CV death (HR: $4.29,2.33$, and 1.03, respectively); BB, ACEI, and statin use could reduce the risk of $\mathrm{CV}$ death (HR:0.53, 0.42, and 0.41, respectively). Finally, smoking and BB use were associated with repeated PCI (HR:1.57 and 1.40, respectively).

Table 5 lists the incidence of adverse outcomes according to the different CKD stages. We found that patients with advanced-stage CKD (stage 4 and 5) had higher cardiovascular mortality and all-cause mortality than those with early-stage CKD (both $P<0.001$ ). However, there was no difference in terms of recurrent MI or repeated PCI $(P=0.06$ and $P=0.20$, respectively). 
Table 2 Demographic Characteristics of the Study Population and Medication Use after the First PCI

\begin{tabular}{|c|c|c|c|c|c|}
\hline \multirow[t]{2}{*}{ Variable } & \multicolumn{4}{|l|}{ Study Group } & \multirow[t]{2}{*}{$P$ value } \\
\hline & Control $(N=509)$ & DM Alone $(N=254)$ & CKD Alone $(N=320)$ & $\mathrm{DM}$ and $\mathrm{CKD} N=311)$ & \\
\hline Gender & & & & & $<0.01$ \\
\hline $\mathrm{F}$ & $87(17.1 \%)$ & $60(23.6 \%)$ & 99 (30.9\%) & $120(38.6 \%)$ & \\
\hline M & $422(82.9 \%)$ & $194(76.4 \%)$ & $221(69.1 \%)$ & $191(61.4 \%)$ & \\
\hline Hypertension & & & & & $<0.01$ \\
\hline No & $265(52.1 \%)$ & $103(40.6 \%)$ & $120(37.5 \%)$ & $91(29.3 \%)$ & \\
\hline Yes & $244(47.9 \%)$ & $151(59.4 \%)$ & $200(62.5 \%)$ & $220(70.7 \%)$ & \\
\hline Hypercholesterolemia & & & & & $<0.01$ \\
\hline No & $189(37.1 \%)$ & 115 (45.3\%) & $139(43.4 \%)$ & $178(57.2 \%)$ & \\
\hline Yes & $320(62.9 \%)$ & 139 (54.7\%) & $181(56.6 \%)$ & $133(42.8 \%)$ & \\
\hline Current smoker & & & & & $<0.01$ \\
\hline No & $269(52.8 \%)$ & $165(65.0 \%)$ & $202(63.3 \%)$ & $237(76.2 \%)$ & \\
\hline Yes & $240(47.2 \%)$ & 89 (35.0\%) & $118(36.7 \%)$ & $74(23.8 \%)$ & \\
\hline Ml & & & & & 0.07 \\
\hline No & $349(68.5 \%)$ & $170(66.9 \%)$ & $191(59.7 \%)$ & $202(65.0 \%)$ & \\
\hline Yes & $160(31.5 \%)$ & $84(33.1 \%)$ & $129(40.3 \%)$ & 109 (35.0\%) & \\
\hline Stroke history & & & & & $<0.01$ \\
\hline No & $492(96.7 \%)$ & $242(95.3 \%)$ & $297(92.8 \%)$ & $283(91.0 \%)$ & \\
\hline Yes & 17 (3.3\%) & $12(4.7 \%)$ & $23(7.2 \%)$ & $28(9.0 \%)$ & \\
\hline CABG history & & & & & 0.06 \\
\hline No & $509(100.0 \%)$ & $252(99.2 \%)$ & 317 (99.1\%) & $306(98.4 \%)$ & \\
\hline Yes & $0(0.0 \%)$ & $2(0.8 \%)$ & $3(0.9 \%)$ & $5(1.6 \%)$ & \\
\hline Asprin & & & & & $<0.01$ \\
\hline No & $25(4.9 \%)$ & $15(5.9 \%)$ & $43(13.4 \%)$ & 37 (11.9\%) & \\
\hline Yes & $484(95.1 \%)$ & 239 (94.1\%) & $277(86.6 \%)$ & 274 (88.1\%) & \\
\hline P2Y12 inhibitors & & & & & 0.72 \\
\hline No & $90(17.7 \%)$ & 43 (16.9\%) & 47 (14.7\%) & $50(16.1 \%)$ & \\
\hline Yes & $419(82.3 \%)$ & 211 (83.1\%) & $273(85.3 \%)$ & 261 (83.9\%) & \\
\hline Diuretics & & & & & $<0.01$ \\
\hline No & $435(85.5 \%)$ & 189 (74.4\%) & $234(73.1 \%)$ & $229(73.6 \%)$ & \\
\hline Yes & 74 (14.5\%) & 65 (25.6\%) & 86 (26.9\%) & 82 (26.4\%) & \\
\hline Beta B & & & & & 0.31 \\
\hline No & $283(55.6 \%)$ & $130(51.2 \%)$ & 189 (59.1\%) & 175 (56.3\%) & \\
\hline Yes & $226(44.4 \%)$ & $124(48.8 \%)$ & $131(40.9 \%)$ & 136 (43.7\%) & \\
\hline $\mathrm{CCB}$ & & & & & 0.37 \\
\hline No & $356(69.9 \%)$ & $164(64.6 \%)$ & 227 (70.9\%) & $212(68.2 \%)$ & \\
\hline Yes & $153(30.1 \%)$ & 90 (35.4\%) & 93 (29.1\%) & 99 (31.8\%) & \\
\hline ACEl & & & & & 0.02 \\
\hline No & $396(77.8 \%)$ & 185 (72.8\%) & $244(76.3 \%)$ & 259 (83.3\%) & \\
\hline Yes & $113(22.2 \%)$ & $69(27.2 \%)$ & $76(23.8 \%)$ & $52(16.7 \%)$ & \\
\hline ARB & & & & & 0.18 \\
\hline No & $401(78.8 \%)$ & $193(76.0 \%)$ & $246(76.9 \%)$ & $224(72.0 \%)$ & \\
\hline Yes & $108(21.2 \%)$ & 61 (24.0\%) & 74 (23.1\%) & 87 (28.0\%) & \\
\hline Statin & & & & & $<0.01$ \\
\hline
\end{tabular}


Table 2 Demographic Characteristics of the Study Population and Medication Use after the First PCl (Continued)

\begin{tabular}{cllll}
\hline No & $281(55.2 \%)$ & $160(63.0 \%)$ & $222(69.4 \%)$ & $234(75.2 \%)$ \\
Yes & $228(44.8 \%)$ & $94(37.0 \%)$ & $98(30.6 \%)$ & $77(24.8 \%)$ \\
Fibrate & & & \\
No & $472(92.7 \%)$ & $229(90.2 \%)$ & $306(95.6 \%)$ & $294(94.5 \%)$ \\
Yes & $37(7.3 \%)$ & $25(9.8 \%)$ & $14(4.4 \%)$ & $17(5.5 \%)$ \\
\hline
\end{tabular}

$D M$ alone diabetes alone, CKD alone chronic kidney disease alone, DM and CKD both DM and CKD, previous $M I$ history of previous myocardial infarction, CABG history history of coronary artery bypass graft, $P 2 Y 12$ inhibitor $\mathrm{P} 2 \mathrm{Y} 12$ receptor inhibitor of platelets, $B B$ beta-blockers, $C C B$ calcium channel blocker, $A C E I$ angiotensin-converting enzyme inhibitor, $A R B$ angiotensin receptor blocker

Table 3 Angiographic Findings and Outcomes

\begin{tabular}{|c|c|c|c|c|c|}
\hline \multirow[t]{2}{*}{ Variable } & \multicolumn{4}{|l|}{ Study Group } & \multirow[t]{2}{*}{$P$ value } \\
\hline & $\begin{array}{l}\text { Control } \\
(N=509)\end{array}$ & $\begin{array}{l}\text { DM alone } \\
(N=254)\end{array}$ & CKD alone $(N=320)$ & $\mathrm{DM}$ and $\mathrm{CKD}(\mathrm{N}=311)$ & \\
\hline Follow-up time (months) & $31.7 \pm 22.6$ & $31.1 \pm 22.4$ & $23.8 \pm 19.7$ & $23.0 \pm 19.2$ & 0.08 \\
\hline Number of diseased vessel & & & & & $<0.01^{*}$ \\
\hline Single-vessel disease & 297 (58.4\%) & $120(47.3 \%)$ & $146(45.6 \%)$ & $106(34.1 \%)$ & \\
\hline Double-vessel disease & $138(27.1 \%)$ & $75(29.5 \%)$ & $90(28.1 \%)$ & $112(36.0 \%)$ & \\
\hline Triple-vessel disease & $74(14.5 \%)$ & $59(23.2 \%)$ & $84(26.3 \%)$ & $93(29.9 \%)$ & \\
\hline Mean treated vessels ${ }^{a}$ & $1.2 \pm 0.4$ & $1.3 \pm 0.5$ & $1.3 \pm 0.5$ & $1.3 \pm 0.5$ & $<0.01^{*}$ \\
\hline Mean treated lesions & $1.4 \pm 0.7$ & $1.5 \pm 0.8$ & $1.5 \pm 0.8$ & $1.6 \pm 0.9$ & 0.07 \\
\hline Type of intervention & & & & & 0.34 \\
\hline Balloon angioplasty (Yes) & $163(32.0 \%)$ & $72(28.4 \%)$ & $109(34.1 \%)$ & $109(35.1 \%)$ & \\
\hline BMS deployment (Yes) & $210(41.3 \%)$ & 87 (34.3\%) & $150(46.9 \%)$ & $130(41.8 \%)$ & \\
\hline DES deployment (Yes) & $183(36.0 \%)$ & $120(47.2 \%)$ & $106(33.1 \%)$ & $116(37.3 \%)$ & \\
\hline Lesion location & & & & & 0.31 \\
\hline$\angle A D$ & $358(70.3 \%)$ & $182(71.7 \%)$ & $236(73.8 \%)$ & $249(80.0 \%)$ & \\
\hline LCX & $223(43.8 \%)$ & 125 (49.2\%) & $160(50.0 \%)$ & $197(63.3 \%)$ & \\
\hline RCA & $218(42.8 \%)$ & $125(49.2 \%)$ & $186(58.1 \%)$ & $182(58.5 \%)$ & \\
\hline SYNTAX score & $9.8 \pm 7.2$ & $11.8 \pm 8.3$ & $11.0 \pm 7.7$ & $11.2 \pm 8.1$ & 0.12 \\
\hline LVEF & $0.61 \pm 0.12$ & $0.59 \pm 0.14$ & $0.58 \pm 0.14$ & $0.55 \pm 0.15$ & $<0.01^{*}$ \\
\hline Ml & & & & & 0.19 \\
\hline Yes & $16(3.1 \%)$ & $13(5.1 \%)$ & $12(3.8 \%)$ & 19 (6.1\%) & \\
\hline No & 493 (96.9\%) & 241 (94.9\%) & 308 (96.2\%) & 292 (93.9\%) & \\
\hline CV death & & & & & $<0.01^{*}$ \\
\hline Yes & $16(3.1 \%)$ & $9(3.5 \%)$ & $27(8.4 \%)$ & $44(14.2 \%)$ & \\
\hline No & 493 (96.9\%) & 245 (96.5\%) & $293(91.6 \%)$ & 267 (85.8\%) & \\
\hline All-cause death & & & & & $<0.01^{*}$ \\
\hline Yes & $20(3.9 \%)$ & 15 (5.9\%) & 49 (15.3\%) & 57 (18.3\%) & \\
\hline No & 489 (96.1\%) & 239 (94.1\%) & $271(84.7 \%)$ & $254(81.7 \%)$ & \\
\hline $\mathrm{Re}-\mathrm{PCl}$ & & & & & 0.87 \\
\hline Yes & 127 (25.0\%) & $63(24.8 \%)$ & 72 (22.5\%) & $74(23.8 \%)$ & \\
\hline No & $382(75.1 \%)$ & 191 (75.2\%) & 248 (77.5\%) & 237 (76.2\%) & \\
\hline
\end{tabular}

BMS bare metal stent, DES drug-eluting stent, LAD left anterior descending artery, LCX left circumflex artery, RCA right coronary artery, SYNTAX score Synergy between Percutaneous Coronary Intervention with Taxus and Cardiac Surgery score, LVEF left ventricular ejection fraction, $\mathrm{MI}$ myocardial infarction, Re-PCI repeated percutaneous coronary intervention

: significant

a : control vs DM alone: $P=0.0059$, control vs CKD alone: $P=0.0027$, control vs DM and CKD: $P=0.0089, \mathrm{DM}$ vs CKD: $P=0.7603, \mathrm{DM}$ vs $\mathrm{DM}$ and CKD: $P=0.7070$, CKD vs DM and CKD: $P=0.4160$ 

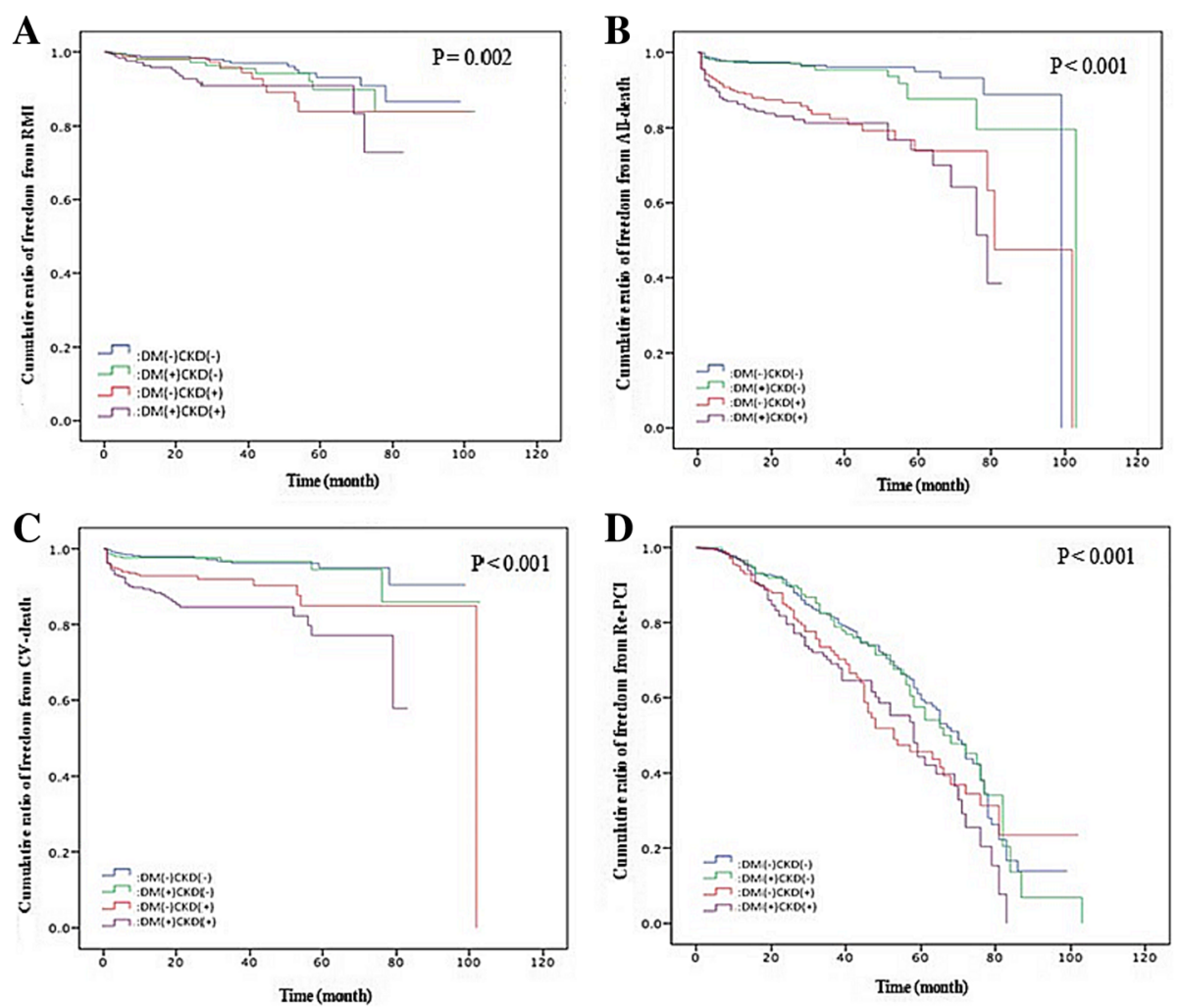

Fig. 1 a Cumulative ratio of freedom from recurrent Ml among the 4 groups $(P=0.002)$. $\mathbf{b}$ Cumulative ratio of freedom from all death among the 4 groups $(P<0.001)$. c Cumulative ratio of freedom from $C V$ death among the 4 groups $(P<0.001)$. $\mathbf{d}$ Cumulative ratio of freedom from Re-PCl among the 4 groups $(P<0.001)$

\section{Discussion}

In patients with $\mathrm{CAD}$ undergoing $\mathrm{PCI}$, we found that the highest rate of all-cause mortality, CV mortality, myocardial infarction, and repeated PCI occurred in patients with both DM and CKD, as compared with patients without DM and CKD, patients with DM alone, and patients with CKD alone. Patients with CKD alone had the second highest risk of all-cause mortality, cardiovascular mortality, and repeated PCI. As for the outcome analysis, we found that smoking and $\mathrm{BB}$ use was associated with repeated PCI procedures. Previous MI and Syntax scores were predictors of MI, and statin use could reduce the risk of MI. Age, previous MI, stroke history, and Syntax score were predictors of allcause death, and BB and statin use could reduce the risk. Previous MI, P2Y12 inhibitor use, and Syntax score were all predictors of $\mathrm{CV}$ death, and $\mathrm{BB}, \mathrm{ACEI}$, and statin use could reduce the risk of $\mathrm{CV}$ death.

In our study, patients with CKD alone, along with patients with both DM and CKD, were older and had a lower BMI; this is compatible with a previous study [17]. Patients with both DM and CKD had a more elevated serum creatinine level than patients with CKD alone $(P<0.001)$, implying that DM had an adverse impact on renal function in CKD patients. Secondarily, patients with both DM and CKD had the highest CSP and lowest CDP, thus they had the most elevated central pulse pressure (CPP). Elevated CPP is strongly associated with adverse cardiovascular outcomes in patients with hypertension patients and in patients after undergoing a PCI procedure in a previous study $[18,19]$. Elevated CPP in patients with DM and CKD may reflect that they had the most advanced arterial stiffness among the 4 groups.

Patients with both DM and CKD had a higher prevalence rate of hypertension than patients with isolated DM or isolated CKD (both $P<0.05$ ), suggesting that $D M$ and CKD had an additive effect on the acceleration of hypertension. In contrast, compared with patients with DM alone or CKD alone, patients with both DM and CKD used statins less frequently as they had the lowest serum cholesterol and LDL-C levels. Similarly, ACEI usage was also the least since patients with both $\mathrm{DM}$ and $\mathrm{CKD}$ had the poorest renal function and concern of increased hazard of hyperkalemia. When a patient has an LDL of $<70 \mathrm{mg} / \mathrm{dL}$, statin use has been found to improve cardiovascular outcomes in CAD patients after ACS [20]; however, whether statin underusage led to the poorest outcome in patients with both DM and CKD in this study remains to be clarified. 
Table 4 Significant Outcome Predictors in a Cox proportion hazard model for recurrent MI,All Death, CV Death, and Repeated PCI

\begin{tabular}{|c|c|c|c|c|}
\hline \multirow[t]{2}{*}{ Variable } & $\mathrm{RMI}^{\mathrm{a}}$ & All-cause death ${ }^{b}$ & CV-death ${ }^{c}$ & Repeated $\mathrm{PCl}^{d}$ \\
\hline & $\mathrm{HR}^{\mathrm{a}}(95 \% \mathrm{Cl})$ & $\mathrm{HR}^{\mathrm{a}}(95 \% \mathrm{Cl})$ & $\mathrm{HR}^{\mathrm{a}}(95 \% \mathrm{Cl})$ & $\mathrm{HR}^{\mathrm{a}}(95 \% \mathrm{Cl})$ \\
\hline \multicolumn{5}{|l|}{ Group } \\
\hline Control & 1.00 (Reference) & 1.00 (Reference) & 1.00 (Reference) & 1.00 (Reference) \\
\hline DM alone & $1.43(0.67-3.06)$ & $1.18(0.59-2.36)$ & $0.91(0.40-2.09)$ & $1.11(0.82-1.52)$ \\
\hline CKD alone & $1.47(0.71-3.08)$ & $2.04(1.15-3.63)^{*}$ & $2.13(1.13-4.01)^{*}$ & $1.47(1.09-1.97)^{*}$ \\
\hline DM and CKD & $2.43(1.23-4.08)^{*}$ & $3.25(1.89-5.59)^{* *}$ & $3.58(1.97-6.49)^{* *}$ & $1.79(1.33-2.41)^{* *}$ \\
\hline Age & - & $1.04(1.02-1.05)^{* *}$ & - & - \\
\hline Smoking & - & - & - & $1.57(1.25-1.96)^{* *}$ \\
\hline Previous Ml & $3.14(1.82-5.40)^{* *}$ & $3.95(2.72-5.74)^{* *}$ & $4.29(2.71-6.79)^{* *}$ & - \\
\hline Stroke & $1.88(0.75-4.74)$ & $1.97(1.16-3.36)^{*}$ & $1.72(0.91-3.25)$ & - \\
\hline Diuretics & - & - & $1.35(0.85-2.15)$ & - \\
\hline DES & - & - & - & - \\
\hline Asprin & & - & $1.33(0.66-2.69)$ & - \\
\hline P2Y12 inh & & - & $2.33(1.01-5.39)^{*}$ & - \\
\hline BB & - & $0.61(0.43-0.88)^{* *}$ & $0.53(0.34-0.83)^{* *}$ & $1.40(1.23-1.74)^{* *}$ \\
\hline$C C B$ & - & - & - & - \\
\hline ACEI & - & - & $0.42(0.25-0.73)^{* *}$ & - \\
\hline ARB & - & - & - & - \\
\hline Statin & $0.46(0.26-0.84)^{*}$ & $0.37(0.23-0.58)^{* *}$ & $0.41(0.24-0.69)^{* *}$ & - \\
\hline Syntax & $1.03(1.01-1.06)^{*}$ & $1.03(1.01-1.04)^{* *}$ & $1.03(1.01-1.05)^{* *}$ & $1.00(0.98-1.01)$ \\
\hline
\end{tabular}

DM alone diabetes alone, CKD alone chronic kidney disease alone, estimated glomerular filtration rate $<60 \mathrm{~mL} / \mathrm{min} ;$ Previous $\mathrm{MI}$ history of previous myocardial infarction, DES drug-eluting stent, $P 2 Y 12$ inh P2Y12 receptor inhibitor of platelets, $B B$ beta-blockers, $C C B$ calcium channel blocker, $A C E l$ angiotensin-converting enzyme inhibitor, $A R B$ angiotensin receptor blocker, Syntax score Synergy between Percutaneous Coronary Intervention with Taxus and Cardiac Surgery score ${ }^{*} P<0.05,{ }^{* *} P<0.01$

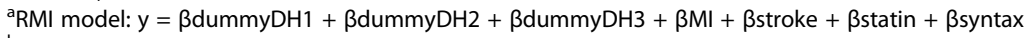

${ }^{\mathrm{b}}$ All-death model: $y=\beta$ dummyDH $1+\beta$ dummyDH $2+\beta$ dummyDH3 $+\beta$ age $+\beta C \mathrm{KD}+\beta \mathrm{Ml}+\beta$ stroke $+\beta$ betab $+\beta$ statin $+\beta$ syntax

${ }^{c} \mathrm{CV}$-death model: $\mathrm{y}=\beta$ dummyDH $1+\beta$ dummyDH $2+\beta$ dummyDH $3+\beta \mathrm{Ml}+\beta$ stroke $+\beta$ diuretics $+\beta$ betab $+\beta A C E \mathrm{~A}+\beta$ statin $+\beta$ syntax

${ }^{d}$ Repeated PCI model: $y=\beta$ dummyDH1 $+\beta$ dummyDH $2+\beta$ dummyDH $3+\beta \mathrm{Ml}+\beta$ smoking $+\beta$ betab $+\beta$ syntax

Table 5 Incidence of Adverse Outcomes with Different CKD Stages According to Estimated Glomerular Filtration Rate

\begin{tabular}{|c|c|c|c|c|c|c|}
\hline \multirow[t]{2}{*}{ Outcome } & \multicolumn{5}{|l|}{ CKD Stage } & \multirow[t]{2}{*}{$P$ value } \\
\hline & 1 & 2 & 3 & 4 & 5 & \\
\hline \multicolumn{7}{|l|}{ Ml } \\
\hline Yes & 99 (32.5\%) & $143(31.8 \%)$ & 147 (35.0\%) & 50 (45.9\%) & 43 (39.1\%) & \multirow[t]{2}{*}{0.0552} \\
\hline No & 206 (67.5\%) & 306 (68.2\%) & $273(65.0 \%)$ & 59 (54.1\%) & 67 (60.9\%) & \\
\hline \multicolumn{7}{|l|}{ CV death } \\
\hline Yes & $11(3.6 \%)$ & 14 (3.1\%) & $32(7.6 \%)$ & $21(19.3 \%)$ & 18 (16.4\%) & \multirow[t]{2}{*}{$<0.0001$} \\
\hline No & 294 (96.4\%) & 436 (96.9\%) & 388 (92.4\%) & 88 (80.7\%) & 92 (83.6\%) & \\
\hline \multicolumn{7}{|c|}{ All-cause death } \\
\hline Yes & $16(5.3 \%)$ & 19 (11.4\%) & 48 (11.4\%) & 32 (29.4\%) & $26(23.6 \%)$ & \multirow[t]{2}{*}{$<0.0001$} \\
\hline No & 289 (94.7\%) & 431 (95.8\%) & 372 (88.6\%) & 77 (70.6\%) & 84 (76.4\%) & \\
\hline \multicolumn{7}{|l|}{$\mathrm{Re}-\mathrm{PCl}$} \\
\hline Yes & 66 (21.6\%) & 124 (27.6\%) & $100(23.8 \%)$ & 20 (18.4\%) & $26(23.6 \%)$ & \multirow[t]{2}{*}{0.2029} \\
\hline No & 239 (78.4\%) & $326(72.4 \%)$ & $320(76.2 \%)$ & 89 (81.6\%) & 84 (76.4\%) & \\
\hline
\end{tabular}


Given for lesion location, intervention type such as balloon angioplasty, bare metal stent deployment, or drug-eluting stent deployment, there were no difference. However, patients with both DM and CKD had the highest prevalence of double-vessel disease and triple-vessel disease; they also had the lowest LVEF. Compared with patients with DM alone, patients with both DM and CKD had a significantly higher risk of developing multivessel disease $(P=0.006)$; when compared with patients with CKD alone, patients with both DM and CKD also had a significantly higher risk of developing multi-vessel disease $(P=0.01)$. Combined DM and CKD seemed to have an additive effect on the progression of atherosclerosis and development of multi-vessel disease than patients with isolated DM or isolated CKD. This is in contrast to a previous study that they observed patients with both DM and hypertension did not have a significant risk of developing multi-vessel disease, as compared with patients with DM alone [21]. However, in terms of treated numbers of the vessels and lesions, no difference existed among patients with DM alone, CKD alone, or both DM and CKD $(P=\mathrm{NS})$; thus, dominance of multivessel disease and poor left ventricular function in patients with DM and CKD may affect long-term outcomes in the present study. Beyond conventional risk factors for the development of CAD, other important prognostic indicators to assess in CAD patients include LVEF and number of diseased vessels [22].

In our study, patients with DM and CKD had the highest rate of all-cause mortality and CV mortality. This may be due to patients with both DM and CKD had a higher rate of multi-vessel disease, along with a poorer LVEF, less statin use, and less ACEI use. On the contrary, while comparing patients with DM alone and CKD alone, patients with CKD alone had an increased rate of all-cause mortality and $\mathrm{CV}$ mortality than patients with $\mathrm{DM}$ alone $(P<0.001$ and $P=0.02$, respectively). However, there were no significant differences between the 2 groups regarding the number of diseased vessels, lesion complexity, LVEF, and medications. In this study, patients with CKD seemed had a poorer outcome than patient with DM. As been stated, a combination of insulin resistance and endothelial dysfunction leads to the progression of atherosclerosis in patients with DM and patients with CKD $[23,24]$; a larger scale clinical trial is necessitated to determine whether patients with CKD have more advanced insulin resistance and endovascular dysfunction than patients with DM.

In conclusion, we found that patients with DM and CKD had the highest mortality after PCI than isolated DM or isolated CKD.; DM and CKD had an additive effect on long-term risks. In terms of adverse outcomes, patients with CKD seemed more hazardous than patients with DM.

\section{Conclusions}

DM and CKD had additive effect on adverse long-term outcomes in patients with.

CAD after receiving PCI. In patients receiving PCI, CKD was a more hazardous outcome predictor than DM.

\section{Study limitations}

First, were did not fully evaluated the intensity of medication, such as tight blood glucose control or blood pressure control. Second, data entry bias may exist; functional evaluations of the atherosclerotic lesions such as fraction flow reserve (FFR) measurement, were not used that may have had an impact on the decision of index PCI enrollment; it has been reported that DM and CKD were independent predictors for FFR measurement. Besides, positive findings of FFR were lower in patients with CKD whereas index of microcirculatory resistance (IMR) was higher [25].Third, since the number of patients in the DM alone group was fewer than that of the other groups, that may have affected the power of this study. Fourth, since DES implantation had a lower adverse cardiac events rate in patients with diabetes or CKD than BMS implantation, patient selection bias may exist in this study.

\section{Abbreviations}

ACEl: Angiotensin-converting enzyme inhibitor; ARB: Angiotensin receptor blocker.; BB: Beta-blockers; BMI: Body mass index; BMS: Bare-metal stent; CABG: Coronary artery bypass graft; CAD: Coronary artery disease;

CAP: Central aortic pressure; CCB: Calcium channel blockers; CDP: Central aortic diastolic pressure; CKD: Chronic kidney disease; CSP: Central aortic systolic pressure; CV Mortality: cardiovascular mortality; DES: Drug-eluting stent; eGFR: Estimated glomerular filtration rate; FFR: Fraction flow reserve; HbA1c: Hemoglobin A1C; HDL-C: High-density lipoprotein-cholesterol; LAD: Left anterior descending artery; Lcx: Left circumflex artery; LDL-C: Lowdensity lipoprotein- cholesterol; LVEF: Left ventricular ejection fraction; MI: Myocardial infarction; OPD: Outpatient department; PCl: Percutaneous coronary intervention; RCA: Right coronary artery; SYNTAX score: Synergy between percutaneous coronary intervention with taxus and cardiac surgery score; TG: Triglyceride

\section{Acknowledgements}

None.

\section{Funding}

This study was supported by a grant from the Department of Research, Taichung Tzu Chi Hospital, Taiwan.

\section{Availability of data and materials}

The data that support the findings of this study are available from the corresponding author on reasonable request.

\section{Authors' contributions}

MJL and HPW conceived and designed the study. MJL collected the study data. JL and CYC analyzed and interpreted the data. CCH performed the statistical analysis. MJ L drafted the manuscript, and HPW revised the manuscript. All authors had read and approved the final manuscript.

Ethics approval and consent to participate

The study protocol was approved by the Institution Review Board and ethics committee of Taichung Tzu Chi Hospital, Taiwan (REC104-19) and written inform consents were obtained from all participants. 


\section{Consent for publication}

Not applicable.

\section{Competing interests}

The authors declare that they have no competing interest.

\section{Publisher's Note}

Springer Nature remains neutral with regard to jurisdictional claims in published maps and institutional affiliations.

\section{Author details}

'Division of Cardiology, Department of Medicine, Taichung Tzu Chi Hospital, The Buddhist Tzu Chi Medical foundation, Taichung, Taiwan. ${ }^{2}$ Department of Medicine, School of Medicine, Tzu Chi University, Hualien, Taiwan. ${ }^{3}$ Division of Pediatric General Medicine, Department of Pediatrics, Chang Gung Memorial Hospital at Linko, No. 5, Fu-Hsin Street, Kweishan, Taoyuan, Taiwan. ${ }^{4}$ College of Medicine, Chang Gung University, Taoyuan, Taiwan. ${ }^{5}$ Department of Pediatric Emergency Medicine, Changhua Christian Children's Hospital, Changhua, Taiwan. ${ }^{6}$ School of Medicine, Kaohsiung Medical University, Kaohsiung, Taiwan. ${ }^{7}$ Department of Public Health, Chung Shan Medical University, Taichung, Taiwan.

Received: 27 April 2017 Accepted: 27 August 2017

\section{Published online: 11 September 2017}

\section{References}

1. Mathew V, Gersh BJ, Williams BA, Laskey WK, Willerson JT, et al. Outcomes in patients with diabetes mellitus undergoing percutaneous coronary intervention in the current era: a report from the prevention of REStenosis with Tranilast and its outcomes (PRESTO) trial. Circulation. 2004;109:476-80.

2. Laskey WK, Seizer F, Viachos HA, Johnston J, Jacobs A, et al. Comparison of in-hospital and one-year outcomes in patients with and without diabetes mellitus undergoing percutaneous catheter intervention (from the national, heart, lung, and blood institute dynamic registry). Am J Cardiol. 2002;90:1062-7.

3. Best PJ, Berger PB, Davis BR, Grines CL, Sadeghi HM, PRESTO Investigators. Impact of mild or moderate chronic kidney disease on the frequency of restenosis: results from the PRESTO trial. J Am Coll Cardiol. 2004:44:1786-91.

4. Norhammar A, Lagerqvist B, Saleh $\mathrm{N}$. Long-term mortality after $\mathrm{PCl}$ in patients with diabetes mellitus: results from the Swedish coronary angiography and angioplasty registry. Eurolntervention. 2010;5:891-7.

5. Kaya E, Cuneo A, Hochadel M, Jünger C, Stepper W, et al. Impact of chronic kidney disease on the prognosis of patients undergoing percutaneous coronary interventions using drug-eluting stents. Clin Res Cardiol. 2011;100:1103-9.

6. Ix JH, Mercado N, Schlipak MG, Lemos PA, Boersma E, et al. Association of chronic kidney disease with clinical outcomes after coronary revascularization: the arterial revascularization therapies study (ARTS). Am Heart J. 2005;149:512-9.

7. Gruberg L, Dangas G, Mehran R, Mintz GS, Kent KM, et al. Clinical outcome following percutaneous coronary interventions in patients with chronic renal failure. Catheter Cardiovasc Interv. 2002:55:66-72.

8. Saltzman AJ, Stone GW, Claessen BE, Narula A, Leon-Reyes S, et al. Longterm impact of chronic kidney disease in patients with ST-segment elevation myocardial infarction treated with primary percutaneous coronary intervention: the HORIZONS-AMI (harmonizing outcomes with revascularization and stents in acute myocardial infarction) trial. JACC Cardiovasc Interv. 2011;4:1011-9.

9. Hanna EB, Chen AY, Roe MT, Wiviott SD, Fox CS, et al. Characteristics and inhospital outcomes of patients with non-ST-segment elevation myocardial infarction and chronic kidney disease undergoing percutaneous coronary intervention. JACC Cardiovasc Interv. 2011:4:1002-8.

10. Klempfner R, Elis A, Matezky S, Keren G, Roth A, et al. Temporal trends in management and outcome of diabetic and non-diabetic patients with acute coronary syndrome (ACS): residual risk of long-term mortality persists: insights from the ACS Israeli survey (ACSIS) 20002010. Int J Cardiol. 2015;179:546-51.

11. Jensen LO, Maeng M, Thayssen P, Tilsted HH, Terkelsen CJ, et al. Influence of diabetes mellitus on clinical outcomes following primary percutaneous coronary intervention in patients with ST-segment elevation myocardial infarction. Am J Cardiol. 2012;109:629-35.
12. Kahn MB, Cubbon RM, Mercer B, Wheatcroft AC, Gherardi G, et al. Association of diabetes with increased all-cause mortality following primary percutaneous coronary intervention for ST-segment elevation myocardial infarction in the contemporary era. Diab Vasc Dis Res. 2012;9:3-9.

13. Goto K, Shiode N, Shirota K, Fukuda Y, Kitamura F, et al. Impact of impaired renal function and diabetes on long-term prognosis in patients undergoing primary angioplasty for acute coronary syndrome. Intern Med. 2008;47:907-13.

14. American Diabetes Association. Diagnosis and classification of diabetes mellitus. Diabetes Care. 2013;36(suppl 1):S67-74.

15. National Kidney Foundation. KDOQI clinical practice guidelines for bone metabolism and disease in chronic kidney disease. Am J Kid Disney Dis. 2003:42:S1-S201.

16. Serruys PW, Morice MC, Kappetein AP, Colombo A, Holmes DR, SYNTAX Investigators. Percutaneous coronary intervention versus coronary-artery bypass grafting for severe coronary artery disease. N Engl J Med. 2009;360: 961-72.

17. limori S, Noda Y, Okado T, Naito S, Toda T, et al. Baseline characteristics and prevalence of cardiovascular disease in newly visiting or referred chronic kidney disease patients to nephrology centers in Japan: a prospective cohort study. BMC Nephrol. 2013;14:152.

18. Williams B, Lacy PS, Thom SM, Cruickshank K, Stanton A, CAFE Investigators. Anglo-Scandinavian Cardia outcomes trial investigators; CAFE steering committee and writing committee. Differential impact of blood pressurelowering drugs on central aortic pressure and clinical outcomes: principal results of the conduit artery function evaluation (CAFE) study. Circulation. 2006:113:1213-25.

19. Lin MJ, Chen CY, Lin HD, Lin CS, Wu HP. Prognostic significance of central pulse pressure for mortality in patients with coronary artery disease receiving repeated percutaneous coronary intervention. Medicine (Baltimore). 2016;95:e3218.

20. Cannon CP, Blazing MA, Giugliano RP, MCCagg A, White JA, IMPROVE-IT Investigators. Ezetimibe added to Statin therapy after acute coronary syndromes. N Engl J Med. 2015;372(25):2387-97.

21. Lin MJ, Chen CY, Lin HD, Wu HP. Impact of diabetes and hypertension on cardiovascular outcomes in patients with coronary artery disease receiving percutaneous coronary intervention. BMC Cardiovasc Disord. 2017:17:12

22. Valgimigli M, Biscaglia S. Stable angina pectoris. Current atherosclero Rep. 2014;16:422

23. Hadi HA, Suwaidi JA. Endothelial dysfunction in diabetes mellitus. Vasc Health Risk Manag. 2007:3:853-76.

24. Chan DT, Watts GF, Irish AB, Dogra GK. Insulin resistance and vascular dysfunction in chronic kidney disease: mechanisms and therapeutic interventions. Nephrol Dial Transplant. 2015;0:1-8.

25. Tebaldi M, Biscaqlia S, Fineschi M, Manari A, Menozzi M, et al. Fractional flow reserve evaluation and chronic kidney disease: analysis from a multicenter Italian registry (the FREAK study). Catheter Cardiovasc Interv. 2016:88(4):555-62

\section{Submit your next manuscript to BioMed Central and we will help you at every step:}

- We accept pre-submission inquiries

- Our selector tool helps you to find the most relevant journal

- We provide round the clock customer support

- Convenient online submission

- Thorough peer review

- Inclusion in PubMed and all major indexing services

- Maximum visibility for your research

Submit your manuscript at www.biomedcentral.com/submit
) Biomed Central 